\title{
A beamformer for CDMA with enhanced near-far resistance
}

\section{Hansen, Henrik; Affes, S.; Mermelstein, P.}

Published in:

IEEE International Conference on Communications, 1999. ICC '99.

Link to article, DOI:

10.1109/ICC.1999.765491

Publication date:

1999

Document Version

Publisher's PDF, also known as Version of record

Link back to DTU Orbit

Citation (APA):

Hansen, H., Affes, S., \& Mermelstein, P. (1999). A beamformer for CDMA with enhanced near-far resistance. In IEEE International Conference on Communications, 1999. ICC '99. (Vol. 3). IEEE.

https://doi.org/10.1109/ICC.1999.765491

\section{General rights}

Copyright and moral rights for the publications made accessible in the public portal are retained by the authors and/or other copyright owners and it is a condition of accessing publications that users recognise and abide by the legal requirements associated with these rights.

- Users may download and print one copy of any publication from the public portal for the purpose of private study or research.

- You may not further distribute the material or use it for any profit-making activity or commercial gain

- You may freely distribute the URL identifying the publication in the public portal

If you believe that this document breaches copyright please contact us providing details, and we will remove access to the work immediately and investigate your claim. 


\title{
A Beamformer for CDMA with Enhanced Near-Far Resistance
}

\author{
Henrik HANSEN ${ }^{1}$, Sofiène AFFES ${ }^{2}$ and Paul MERMELSTEIN ${ }^{2}$ \\ ${ }^{1}$ Dept. of Telecommunication, Tech. Univ. of Denmark, \\ Bldg. 371, DK-2800 Lyngby, Denmark \\ 2 INRS-Télécommunications, Université du Quebec, \\ 16, Place du Commerce, Ile-des-Sours, Verdun, Qc., H3E 1H6, Canada
}

\begin{abstract}
The Spatio-Temporal Array-Receiver (STAR) achieves good performance in CDMA with multiple receiving antennas where the interference can be characterized as AWGN uncorrelated with the signal. To enhance its near-far resistance in correlated noise environments, we introduce optimal combining of the spatio-temporal components. Nearly as good performance can be obtained with a low complexity adaptive beamformer combination of the antenna and multipath diversity branches. Simulation results indicate that the modifled STAR manifests significant gain in near-far resistance over its original version, more so with more branches. They also suggest that exploiting additional temporal correlation fingers as interference references can further improve near-far resistance in poor diversity situations.
\end{abstract}

\section{INTRODUCTION}

The need to improve capacity in wireless mobile communication systems has recently focused research attention on array processing techniques. Array processing was recently applied to synchronous CDMA [1] to improve the capacity of personal communication systems. However, on the uplink the received signal cannot be considered synchronous; and, as a result, receiver structures for asynchronous CDMA $[2,3]$ have been proposed. One promising scheme is the Spatio-Temporal Array-Receiver (STAR) [4]. STAR performs blind identification of the channel using knowledge of the code of the desired user only and joint tracking in space and time with low complexity. Performance in realistic environments merits further attention.

So far, the behavior of STAR has been studied in a frequency selective Rayleigh fading environment under the assumption that the received signal is corrupted by Additive White Gaussian Noise (AWGN). The AWGN assumption is motivated by consideration of the co-channel interference. Assuming many co-existing users jointly power-controlled, the central limit theorem suggests AWGN-like interference. However, we may encounter situations where the white noise assumption is not well justified. For example, in multi-rate CDMA where some users are assigned a low processing gain (e.g., high speed data applications), while others are assigned a high processing gain (e.g., speech), the user with the lower processing gain must use more power to achieve the same $S N R$ after despreading.

In this paper we relax the AWGN assumption and investigate the beamformer behavior in colored noise. In particular, we investigate the impact of a single user transmitting at a much higher power level. This user is referred

This work was supported by the Bell Quebec/Nortel/NSERC Industrial Research Chair in Personal Communications. to below as the jammer.

We introduce a new adaptive beamformer structure to replace the Maximal Ratio Combiner (MRC) used in the original version of STAR [4]. This structure is an adaptive implementation [5] of the optimal beamformer [6], a combiner whose theoretical advantages are already established in wireless communications (see [7] and references therein). The optimal beamformer significantly enhances the nearfar resistance of STAR as confirmed by simulations.

\section{Signal Model AND Description of STAR}

For the sake of completeness, the signal model and the description of STAR are reviewed here. The presentation is very brief, but details are available in [4].

\section{A. Signal Model}

We consider a cellular CDMA system where each basestation is equipped with a receiving antenna of $M$ sensors. We assume the general case of a selective fading environment where the number of paths is denoted by $P$. We also define the processing gain $L$ and use the following data model after despreading, referred to as the postcorrelation model (PCM) [4].

The received signal vector from the antenna array is decorrelated by the code of the desired user, sampled at the chip rate and framed at the bit rate, resulting in the post-correlation observation matrix $\mathbf{Z}_{n}$ :

$$
\mathbf{Z}_{n} \simeq b_{n} \psi_{n} \mathbf{G}_{n} \Upsilon_{n} \mathbf{D}_{n}^{T}+\mathbf{N}_{n}=b_{n} \psi_{n} \mathbf{H}_{n}+\mathbf{N}_{n}
$$

where $s_{n}=b_{n} \psi_{n}$ is the signal component, $b_{n}$ is the transmitted DBPSK data sequence and $\psi_{n}^{2}$ is the total received power. $\mathbf{G}_{n} \in \mathbb{C}^{M \times P}$ is the steering matrix, $\Upsilon_{n} \in \mathbb{C}^{P \times P}$ is a diagonal matrix of power ratios summing to 1 over all paths, $\mathbf{D}_{n} \in \mathbb{C}^{L \times P}$ is a matrix whose columns are replicas of the correlation function of the chip shaping pulse, each time-shifted by one of the $P$ multipath delays $\tau_{1}, \ldots, \tau_{P}$. Finally, the total spatio-temporal channel-response matrix is given by $\mathbf{H}_{n} \in \mathbb{C}^{M \times L}$ and $\mathrm{N}_{n} \in \mathbb{C}^{M \times L}$ is the spatiotemporal noise matrix. We abandon here the previous assumption that it is uncorrelated [4].

The matrices $\mathbf{Z}_{n}, \mathbf{H}_{n}$ and $\mathbf{N}_{n}$ are transformed into $(M L)$-dimensional vectors by concatenating their columns, leaving one spatio-temporal column vector:

$$
\underline{\mathrm{Z}}_{n}=\underline{\mathrm{H}}_{n} s_{n}+\underline{\mathrm{N}}_{n}
$$

where $\underline{\mathrm{Z}}_{n}, \underline{\mathrm{H}}_{n}$, and $\underline{\mathrm{N}}_{n}$ denote the resulting vectors. 


\section{B. Description of STAR}

STAR performs acquisition and tracking. The demodulation of the received signal is initiated by acquisition which involves identification of the channel; that is, an estimate of the number of paths, $\hat{P}$, and their time-delays. Details can be found in [4]. Once the signal has been acquired, the channel is iteratively estimated in the tracking mode briefly described below.

Suppose an estimate of $\underline{\hat{H}}_{n}$ is available. Then an estimate of the signal component, $\hat{s}_{n}$, is obtained as:

$$
\hat{s}_{n}=\operatorname{Real}\left\{\underline{\mathrm{W}}_{n}^{m r H} \underline{\mathrm{Z}}_{n}\right\}
$$

where $\underline{\mathrm{W}}_{n}^{m r}=\underline{\hat{\mathrm{H}}}_{n} /\left(\underline{\hat{\mathrm{H}}}_{n}^{H} \underline{\hat{\mathrm{H}}}_{n}\right)=\underline{\hat{\mathrm{H}}}_{n} / M$ is the MRC beamformer. This beamformer, which has been used in STAR, promises optimality in the case of uncorrelated white noise.

Using the estimated signal component $\hat{\boldsymbol{s}}_{n}, \underline{\mathrm{H}}_{n}$ is updated at the bit rate in two steps. First, $\underline{\tilde{\mathrm{H}}}_{n+1}$ is computed in the following LMS-type subspace tracking procedure [4]:

$$
\underline{\tilde{H}}_{n+1}=\underline{\hat{H}}_{n}+\mu\left(\underline{\mathrm{Z}}_{n}-\underline{\hat{\mathrm{H}}}_{n} \hat{\mathrm{s}}_{n}\right) \hat{\mathrm{s}}_{n}^{H} \text {, }
$$

where $\mu$ is an adaptation step-size. Next, the time-delay estimates are updated in a tracking algorithm. The estimated time-delays determine $\hat{\mathbf{D}}_{n}$, which is used to refine the estimate of $\underline{\tilde{\mathrm{H}}}_{n+1}$ considerably using structure fitting, providing the result $\underline{\hat{H}}_{n+1}[4]$.

\section{Optimal Beamforming Structures}

In this section we propose optimal beamforming structures to replace the MRC beamformer used in the classical version of STAR [4]. To provide a general expression for optimal beamforming [6], we exploit in a first version all the temporal-correlation dimension $L$ of the post-correlation data. To save computations in a second version, we reduce this dimension to the number of paths $P$ at the cost of some loss in interference rejection and near-far resistance. In other versions with intermediate dimensions, we achieve a better tradeoff between these extreme cases. Finally, for practical implementation we propose a general adaptive structure for these beamformers.

\section{A. Exploitation of Temporal Correlation}

As mentioned earlier, MRC is only optimal in the case of white noise. Therefore, we now consider the optimal beamformer for colored noise in the entire spatio-temporal observation space. With the observation model of Eq. (2) used here, the optimal beamformer is given by [6]:

$$
\underline{\mathrm{W}}_{n}^{o p}=\frac{\mathbf{R}_{\underline{\underline{\mathrm{Z}}}}^{-1} \underline{\mathrm{H}}_{n}}{\underline{\mathrm{H}}_{n}^{H} \mathbf{R}_{\underline{\mathrm{Z}}^{-1} \underline{\mathrm{H}}_{n}}}=\frac{\mathbf{R}_{\underline{\underline{\mathrm{N}}}}^{-1} \underline{\mathrm{H}}_{n}}{\underline{\mathrm{H}}_{n}^{H} \mathbf{R}_{\underline{\underline{N}}}^{-1} \underline{\underline{H}}_{n}},
$$

where $\mathbf{R}_{\underline{N}} \in \mathbb{C}^{M L \times M L}$ is the correlation matrix of the noise.

Although Eq. (5) is optimal, its implementation is extremely complex. Its complexity turns out to be proportional to the third power of the spatio-temporal observation-space dimension (i.e., $M^{3} L^{3}$ ) [5]. Therefore, a simpler approach is sought. The idea is to reduce as much as possible the dimension of the observation space, without excluding any of the $M P$ diversity branches available.

Evidently, we obtain the smallest dimension by extracting these $M P$ fingers in a RAKE-like fashion, to yield the alternative observation vector $\underline{Y}_{n} \in \mathbf{C}^{M P \times 1}$ given by:

$$
\underline{Y}_{n}=\underline{J}_{n} s_{n}+\underline{N}_{n}^{\prime} \text {, }
$$

where the steering vector $\underline{J}_{n} \in \mathbb{C}^{M P \times 1}$ holds the $M P$ diversity branches from antennas and multipaths and $\underline{\mathrm{N}}_{n}^{\prime} \in$ $\mathbb{C}^{M P \times 1}$ is the transformed noise vector. $\underline{Y}_{n}$ and $\underline{J}_{n,}$ are obtained from $\mathbf{Z}_{n}$ and $\mathbf{H}_{n}$, respectively, by reshaping columnwise in one vector the following matrices:

$$
\begin{aligned}
\mathbf{Y}_{n}^{T} & =\left(\mathbf{D}_{n}^{T} \mathbf{D}_{n}\right)^{-1} \mathbf{D}_{n}^{T} \mathbf{Z}_{n}^{T}, \\
\mathbf{J}_{n}^{T} & =\left(\mathbf{D}_{n}^{T} \mathbf{D}_{n}\right)^{-1} \mathbf{D}_{n}^{T} \mathbf{H}_{n}^{T},
\end{aligned}
$$

where $\left(\mathbf{D}_{n}^{T} \mathbf{D}_{n}\right)^{-1} \mathbf{D}_{n}^{T}$ is a multi-dimensional temporal MRC beamformer [4] that operates like a finger selector at the multipath time-delays $\tau_{1}, \ldots, \tau_{P}$. Using this model, $\underline{\mathrm{H}}_{n}$ in (5) is replaced by $\underline{J}_{n}$ to arrive at the reduced cornplexity beamformer $\underline{W}_{n}^{r c}$ given by:

$$
\underline{W}_{n}^{r c}=\frac{\mathbf{R}_{\underline{\mathbf{Y}}}^{-1} \underline{J}_{n}}{\underline{J}_{n}^{H} \mathbf{R}_{\underline{Y}}^{-1} \underline{J}_{n}}=\frac{\mathbf{R}_{\underline{\underline{N}}^{\prime} \underline{J}_{n}}^{-1}}{\underline{J}_{n}^{H} \mathbf{R}_{\underline{\underline{N}}^{\prime}}^{-1} \underline{J}_{n}} .
$$

It reduces complexity by a significant factor of $\left(\frac{L}{P}\right)^{3}$.

This beamformer is optimal in the reduced spatiotemporal observation-subspace. However, it is sub-optimal in the entire observation-space. Indeed, although we exploit all diversity branches retained in the obse:vationsubspace, all temporal correlation references or siatistics in the discarded dimensions are excluded, which rnay degrade the interference rejection. We will assess in section IV-A the penalty in interference rejection associated with this approach and will show that it is not severe.

In any case, we can increase the temporal dimersion at the cost of a reasonable increase in complexity s. as to improve interference rejection and near-far resistance. For instance, we may arbitrarily consider not only the timedelays $\tau_{p}$, but also the neighbors $\tau_{p}-T_{c}$ and $\tau_{p}+T_{c}$ in an expanded version of the finger selector $\left(\mathbf{D}_{n}^{T} \mathbf{D}_{n}\right)^{-1} \mathbf{D}_{n}^{T}$, resulting in an observation model similar to Eq. (ij) with the appropriate modified dimensions. We may also develop a selection strategy, but that is beyond the scope of this paper. We show later by simulations that exploiting the temporal correlation alone, following the simple strategy above, improves interference rejection and near-far resistance. We name this approach the eXtra-finger (X-finger) version.

\section{B. Adaptive Implementation}

The beamformers given above can be estimated using sample correlation matrices and channel estimates. However, optimal beamforming is sensitive to estimation errors. Due to the rapid variations of a mobile channel, the noise correlation matrix and the channel-response change with 
time and increase these errors. Therefore, an algorithm which adaptively implements the optimal beamformer is needed. We use below a time-adjusted version [5] of the Generalized Sidelobe Canceller (GSC) structure [8] to implement the reduced complexity beamformer of Eq. (8). Modifications for other beamformer dimensions (see previous section) are ad hoc. The time-adjusted GSC structure is the following [5]:

$$
\begin{aligned}
y_{n} & =\left(\underline{\hat{\mathrm{J}}}_{n}^{H} \underline{\mathrm{J}}_{n}\right)^{-1} \underline{\mathrm{J}}_{n}^{H} \underline{\mathrm{Y}}_{n}, \\
\underline{\mathrm{V}}_{n} & =\mathbf{B}_{n}^{H} \underline{\mathrm{Y}}_{n}, \\
\hat{\mathrm{s}}_{n} & =y_{n}-\underline{\mathrm{W}}_{n}^{i^{H}} \underline{\mathrm{V}}_{n}, \\
\underline{\mathrm{W}}_{n+1}^{i} & =\underline{\mathrm{W}}_{n}^{i}+\eta \underline{\mathrm{V}}_{n} \hat{s}_{n}^{H},
\end{aligned}
$$

where $\mathbf{B}_{n} \in \mathbb{C}^{M P \times M P-1}$ is a signal-blocking matrix satisfying $\mathbf{B}_{n}^{H} \underline{\mathrm{J}}_{n}=\underline{0}_{M P-1}$ and $\mathbf{B}_{n}^{H} \mathbf{B}_{n}=\mathbf{I}_{M P-1}, y_{n}$ is the signal component from the output of the MRC beamformer matched to $\mathrm{J}_{n}, \underline{\mathrm{V}}_{n}$ is the interference-reference vector, $\underline{W}_{n}^{i}$ is the interference cancelling-filter, $\hat{s}_{n}$ is the signal component estimate and $\eta$ is the adaptation step-size [8]. To rotate the noise subspace similarly to the rotation of the signal subspace spanned by $\underline{J}_{n}$, note that the blocking matrix $B_{n}$ must be adaptively updated at every iteration [5]. Fig. 1 shows the old MRC and the new time-adjusted GSC beamforming structures in the block diagram of STAR.

(a)

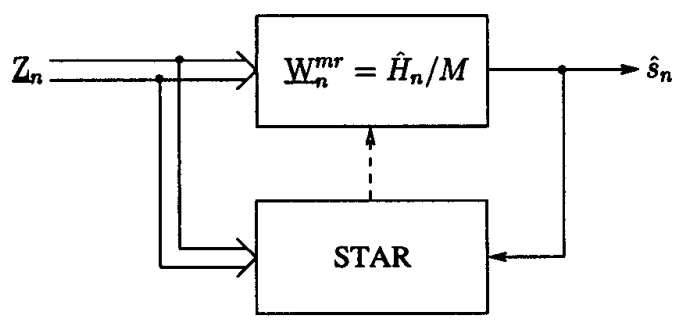

(b)

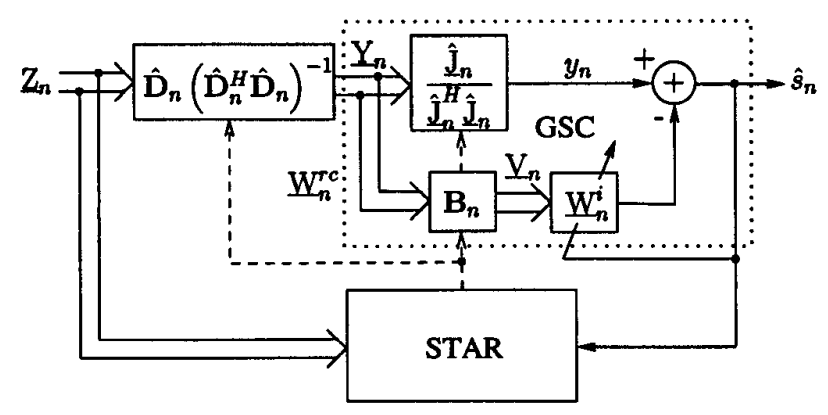

Fig. 1. Beamforming structures in the block diagram of STAR. (a): the old MRC beamformer. (b): the new time-adjusted GSC beamformer.

\section{Simulations}

In section IV-A we compare the performance in Signal to Noise Ratio at the Output (SNRO) of the optimal and reduced complexity beamformers described in the previous section with the MRC version. In section IV-B we compare the performance of STAR in colored noise in terms of $B E R$ and tracking "failure rate" when utilizing the MRC combiner and the GSC implementation of the reduced complexity beamformer.

\section{A. Performance in Output SNR}

The $S N R O$ for the MRC, optimal and reduced complexity beamformers is given by $M^{2} /\left(\underline{\mathrm{H}}_{n}^{H} \mathbf{R} \underline{\underline{\mathrm{H}}} \underline{\underline{H}}_{n}\right), \underline{\mathrm{H}}_{n}^{H} \mathbf{R}_{\underline{\mathrm{N}}}^{-1} \underline{\mathrm{H}}_{n}$ and $\underline{J}_{n}^{H} \mathbf{R}_{\mathbf{N}^{\prime}}^{-1} \underline{J}_{n}$, respectively. These expressions can be computed explicitly, given the spatio-temporal channelresponse $\underline{H}_{n}$ and the noise correlation matrix $\mathbf{R}_{\underline{N}}$ in the entire observation space. In particular, if we assume that the time-delays $\tau_{p}$ are multiples of the chip duration, then all the elements of $\mathbf{R}_{\underline{N^{\prime}}}$ can be found in $\mathbf{R}_{\underline{\underline{N}}}$ at the appropriate indices. $\underline{J}_{n}$ is obtained in (7) from $\underline{\underline{H}}_{n}$. In the case of a single interfering jammer, we can write $\mathbf{R}_{\underline{N}}$ :

$$
\mathbf{R}_{\underline{\underline{N}}}=\mathbf{R}_{\underline{I}}+\sigma_{n}^{2} \mathbf{I}_{M L},
$$

where $\sigma_{n}^{2}$ is the white noise power. $\mathbf{R}_{\underline{I}}$ is the correlation matrix of the interfering jammer. Its spatio-temporal element of double index $\left(k_{1}, k_{2}\right) \in\{1, \ldots, M L\} \times\{1, \ldots, M L\}$ is given by ${ }^{1}$ :

$$
\begin{gathered}
\mathbf{R}_{\underline{I}}\left(k_{1}, k_{2}\right)=E\left\{Z_{k_{1}} Z_{k_{2}}^{H}\right\}= \\
\sum_{n=-1}^{n=1}\left[\sum_{p=1}^{P} \alpha_{p}^{m_{1}} \rho_{c c}\left(n_{1}-n L-\tau_{p}^{i}\right)\right]\left[\sum_{p=1}^{P} \alpha_{p}^{m_{2}} \rho_{c c}\left(n_{2}-n L-\tau_{p}^{i}\right)\right]^{H}
\end{gathered}
$$

where $\alpha_{p}^{m}$ is the channel response of the $p$-th path from the jammer to the $m$-th sensor, $\rho_{c c}(x)$ is the cross-correlation function between the user and the jammer codes (note that $\rho_{c c}(x)=0$ for $\left.|x| \geq L\right)$ and $\tau_{p}^{i} \in\{0 \ldots(L-1)\}$ is the $p$-th path-delay of the jammer.

\begin{tabular}{||c||c|c|c|c|c||}
\hline \hline$\sigma_{i}^{2} /\left(\sigma_{n}^{2}+\sigma_{i}^{2}\right)$ & 0 & 0.3 & 0.5 & 0.7 & 0.9 \\
\hline \hline$S N R O_{m r}[\mathrm{~dB}]$ & 6.00 & 4.68 & 3.48 & 1.89 & -3.03 \\
\hline$S N R O_{o p}[\mathrm{~dB}]$ & 6.00 & 5.92 & 5.92 & 5.91 & 5.90 \\
\hline$S N R O_{r c}[\mathrm{~dB}]$ & 6.00 & 5.57 & 5.45 & 5.34 & 5.19 \\
\hline \hline
\end{tabular}

Tab. 1. SNRO in $\mathrm{dB}$ for various beamformers as a function of the interference power relative to the total noise power.

The $S N R O$ results are listed in Tab. 1 for various values of interference to total noise power ratio $\sigma_{i}^{2} /\left(\sigma_{n}^{2}+\sigma_{i}^{2}\right)$ where $\sigma_{i}^{2}$ is the power of the interfering jammer. They are obtained for a code length $L=32, M=4$ antennas and $M P=12$ independent Rayleigh distributed path gains (i.e., $P=3$ path-delays), after averaging over 1000 different channel responses and noise correlations at an $S N R$ of $0 \mathrm{~dB}$ after despreading. Subscripts ' $m r$ ', 'op' and 'rc' refer to $S N R O$ values for the MRC, optimal and reduced complexity beamformers, respectively.

${ }^{1}\left(k_{1}, k_{2}\right)=\left(n_{1} M+m_{1}, n_{2} M+m_{2}\right)$ where $n_{1}$ and $n_{2} \in\{1 \ldots L\}$ are the chip indices and $m_{1}$ and $m_{2} \in\{1 \ldots M\}$ are the antenna indices. 
As the power of the interfering jammer increases, we observe that the SNRO for MRC in the second row of Tab. 1 drops very quickly from its maximum of $10 \log _{10}(M)=6$ $\mathrm{dB}$ (i.e., antenna gain) achieved in white noise. It shows the severe limitations of the MRC beamformer in colored noise. On the other hand, we note that the $S N R O$ for the optimal beamformer in the third row remains almost constant at $6 \mathrm{~dB}$, showing a significant and stable near-far resistance to an increasing power of the jammer.

In the last row of Tab. 1, the reduced complexity beamformer approaches this performance with a tightly contained degradation of the $S N R O$. Since the optimal beamformer is computationally more complex, the reduced complexity beamformer can be regarded as a very good compromise for practical implementation of STAR with a significant enhancement of its near-far resistance. In the following we shall use the GSC structure to implement this beamformer.

\section{B. Performance in Failure Rate and BER}

The $S N R O$ results given in the previous section are obtained by Monte Carlo simulation of theoretical expressions assuming perfect channel identification and tracking with any of the studied beamformers and in any near-far situation. In practice, channel identification and tracking do not respond equally to the above conditions. Besides, they completely fail in critical near-far situations, more so with less resistant beamformers. The $S N R O$ results do not provide realistic insights of practical beamformer performance and should be complemented by other criteria. In this section we compare the performance of STAR in terms of the tracking failure rate and the $B E R$ over the successful runs (see explanations shortly below) when utilizing the MRC and the GSC beamformers.

We consider both the frequency-selective case with $P=3$ paths of equal strength and the non-selective case (i.e., $P=$ 1). The corresponding $M P$ Rayleigh fading multipaths are generated independently following the model of Jakes [9] for a mobile speed of $5 \mathrm{~km} / \mathrm{h}$ and a carrier frequency of $1.9 \mathrm{GHz}$; resulting in a maximum Doppler of $10 \mathrm{~Hz}$ at a BPSK data rate of $9.6 \mathrm{kbits} / \mathrm{sec}$. We also implement power control as described in [4].

The tested CDMA system consists of a desired user and a jammer which transmits at a power level significantly exceeding that of the desired user. All other users are modeled as AWGN at a nominal $S N R$ value ${ }^{2}$ of $8 \mathrm{~dB}$ after despreading. In each simulation we employ new random codes with a processing gain $L=128$ for both the desired user and the jammer. The initial time-delays of the desired signal and the jammer are chosen randomly with a maximum spread of 14 chips and are all changed linearly at a rate of -2.5 and $2.5 \mathrm{chips} / \mathrm{sec}^{3}$, respectively. The step-size values in Eqs. (4) and (9) are $\mu=0.04$ and $\eta=0.01$, respectively. Finally, the interference cancelling-filter $\underline{W}_{n}^{i}$ is

\footnotetext{
2The actual $S N R$ cannot be fixed because of imperfect power control. However, it deviates only slightly from the nominal one.

${ }^{3}$ This arbitrarily-shaped time-drift can be attributed to mobile relative motion and/or to uncertainty of transmitter and receiver clocks.
}

initialized to $\underline{0}_{M P-1}$, reflecting the critical situation where the jammer appears abruptly at iteration 0 .

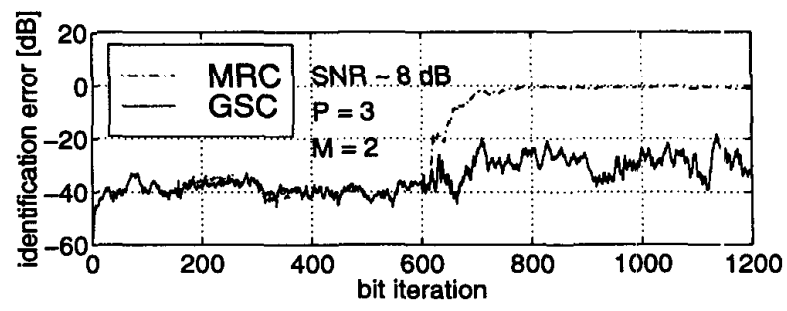

Fig. 2. Channel identification errors of STAR with the MIRC and GSC beamformers at an $S I R$ of $2 \mathrm{~dB}$ with 2 antennas in selective Rayleigh fading.

To gain a better understanding of the tracking failure rate, we show in Fig. 2 channel identification errors (i.e., $\left|1 \pm \underline{\hat{\mathrm{H}}}_{n}^{H} \underline{\mathrm{H}}_{n} / M\right|^{2}$ ) of STAR with the MRC and GSC beamformers at an $S I R$ of $2 \mathrm{~dB}$. The solid curve indicates that the GSC structure is near-far resistant to the jammer during the selected observation interval of 125 msec. On the other hand, the semi-dashed curve shows that MRC performs nearly as well as the GSC until iteration $n \simeq 620$ where the tracking loop breaks (i.e., fails) without any possible relock, resulting in a very low $S N R O$ and a very large $B E R$. In such situations, the link is dropped in practice. We therefore provide for either beamformers the failure rate (i.e., failing runs) and the $B E R$ computed over the successful runs. Many criteria can be applied to determine when tracking fails. For the sake of simplicity, tracking is said to fail in the following if the mean square error of the time-delay estimates over the last 200-iteration ir.terval, $E\left\{(\hat{\tau}-\tau)^{2}\right\}$, is greater than 0.04 (which has been verified to be a proper choice with the $S N R$ and $S I R$ values used in the simulations). (a)

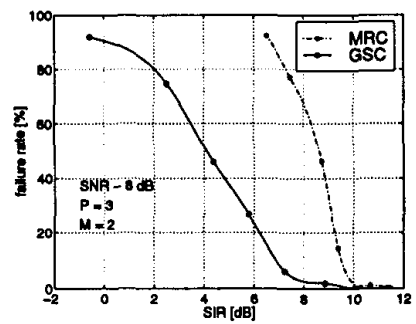

(b)

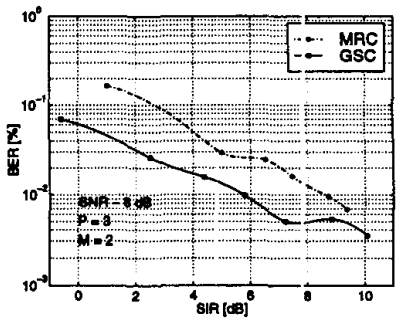

Fig. 3. Performance of MRC and GSC vs. $S I R$ with 2 antinnas in selective Rayleigh fading. (a): failure rate. (b): $B E R$.

Figs. $3 \mathrm{a}$ and $3 \mathrm{~b}$ respectively depict the failure rate and the $B E R$ versus $S I R$ for the MRC and GSC beanformers with $M=2$ antennas in the frequency-selective case (i.e., $P=3$ ). They show that STAR with the GSC gains significantly in near-far resistance compared to the previous version with MRC. In Fig. 3a, the MRC bear.former proves to be robust to an interfering jammer with a minimum $S I R$ of around 10-11 dB whereas the GSC; offers improved robustness to an additional $2 \mathrm{~dB}$ of interference 
power. At a failure rate of $10 \%$, we measure a gain in $S I R$ of the GSC over MRC of about 3-4 dB, which becomes greater at higher failure rates. On the other hand, at a required $B E R$ of $1 \%$ (before FEC decoding), Fig. 3b shows that STAR with the GSC can handle a jamming interferer about 2-3 dB stronger than with MRC. At increasing $S I R$ values approaching the white noise case, both beamformers tend to offer similar performance in $B E R$. (a)

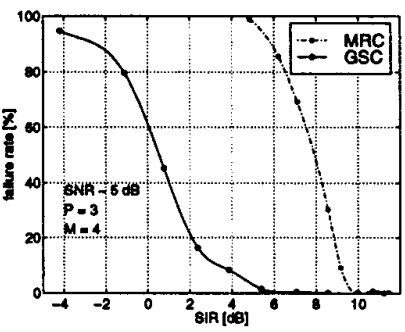

(b)

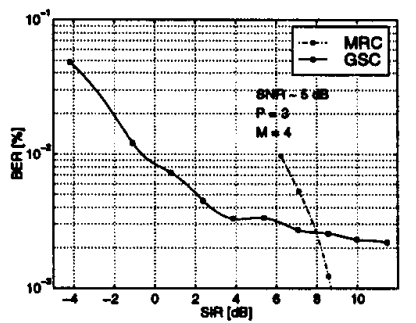

Fig. 4. Performance of MRC and GSC vs. $S I R$ with 4 antennas in selective Rayleigh fading. (a): failure rate. (b): $B E R$.

In Fig. 4, we show that two additional antennas (i.e., $M=4$ ) further improve the interference rejection and nearfar resistance significantly at an $S N R$ of $5 \mathrm{~dB}$ (to obtain results comparable to the two-antenna case). At a failure rate of $10 \%$, we measure in Fig. 4a a gain in $S I R$ of the GSC over MRC of about $6 \mathrm{~dB}$, twice as much as the case with two antennas. In Fig. 4a, the $B E R$ improvement is significant too. At a $B E R$ of $1 \%$, the GSC offers an additional $S I R$ margin as high as $7 \mathrm{~dB}$. At high $S I R$ values approaching the white noise case, note that the perturbation errors due to the unnecessary adaptations of the GSC structure become noticeable and limit its $B E R$ performance compared to MRC. (a)

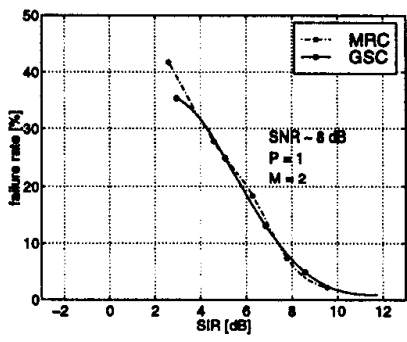

(b)

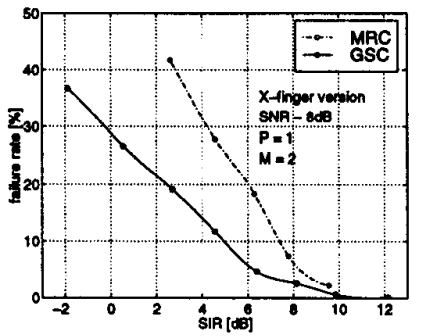

Fig. 5. Failure rate of MRC and GSC vs. SIR with 2 antennas in non-selective Rayleigh fading. (a): GSC implements the reduced complexity beamformer. (b): GSC implements the X-finger version.

In Fig. 5, we briefly study the non-selective case (i.e., $P=1$ ) to show the advantage of exploiting the temporal correlation (see section III-A). Fig. 5a shows that MRC and the GSC implementation of the reduced complexity beamformer offer about the same performance. Clearly, the dimension of the observation subspace exploited by the reduced complexity beamformer of $M P=2$ leaves only one degree of freedom for interference and noise reduction and thus allows only a negligible improvement of near-far resistance. As suggested in section III-A, we can expand this dimension to $3 M P$ in the $\mathrm{X}$-finger version to enhance nearfar resistance without compromising complexity. Clearly, this modified version of the GSC in Fig. 5b offers a noticeable $S I R$ gain over MRC - roughly $2-3 \mathrm{~dB}$ at a failure rate of $10 \%$ - and confirms the advantage of exploiting the temporal correlation to improve near-far resistance.

\section{Conclusions}

To improve the near-far resistance of STAR in colored noise, we replaced the MRC combiner with an optimal beamformer. By theoretical analysis of the output $S N R$, we observe that optimum combining of the antenna and multipath diversity branches performs near as well as optimum beamforming in the entire spatio-temporal observation space. To put this reduced complexity beamformer from theory into practice, we proposed a low complexity time-adjusted implementation of the adaptive GSC beamformer. Simulation results indicate that the original version of STAR is modestly robust to a strong interfering jammer. On the other hand, they show that the time-adjusted GSC structure significantly enhances the near-far resistance of the modified STAR. Finally, they suggest that exploiting additional temporal correlation fingers as interference references can improve near-far resistance in poor diversity situations without compromising complexity. The modified STAR and the adaptive optimum beamformer may find significant applications in CDMA systems supporting multi-rate transmission with different received power levels. They may also reduce the loss due to imperfect power control in equal-rate transmissions.

\section{REFERENCES}

[1] A. J. Paulraj and C. B. Papadias, "Space-time signal processing for wireless communications", IEEE Signal Processing Magazine, vol. 14, no. 6, pp. 49-83, November 1997.

[2] A.F. Naguib and A. Paulraj, "Effects of multipath and basestation antenna arrays on uplink capacity of cellular CDMA", in Proc. GLOBECOM'94, 1994, pp. 395-399.

[3] H. Liu and G. Xu, "Blind equalization for CDMA systems with aperiodic spreading sequences", in Proc. ICASSP'96, vol. 5, 1996, pp. 2658-2661.

[4] S. Affes and P. Mermelstein, "A new receiver structure for asynchronous CDMA: STAR - the spatio-temporal array receiver", IEEE Journal on Selected Areas in Communications, vol. 16, no. 8, pp. 1411-1422, October 1998.

[5] S. Affes, S. Gazor and Y. Grenier, "An algorithm for multisource beamforming and multitarget tracking: further results", in Proc. EURASIP EUSIPCO'96, vol. I, 1996, pp. 543-546.

[6] J. Capon, R. J. Greeenfield, and R. J. Kolker, "Multidimensional maximum-likelihood processing of a large aperture seismic array", Proceedings of the IEEE, vol. 55, pp. 192-211, 1967.

[7] J.H. Winters and J. Salz, "Upper bounds on the bit-error rate of optimum combining in wireless systems", IEEE Transactions on Communications, vol. 46, no. 12, pp. 1619-1624, December 1998.

[8] L. J. Griffiths and C. W. Jim, "An alternative approach to linearly constrained adaptive beamforming", IEEE Transactions on Antennas and Propagation, vol. 30, no. 1, pp. 27-34, January 1982 .

[9] W.C. Jakes, Ed., Microwave Mobile Communications, John Wiley \& Sons, 1974 